\title{
Nosocomial blood-stream infections from extended-spectrum-beta- lactamase-producing Escherichia coli and Klebsiella pneumonia from GB Pant Hospital, New Delhi
}

\author{
Juhi Taneja, Bibhabati Mishra, Archana Thakur, Vinita Dogra, Poonam Loomba \\ Department of Microbiology GB Pant Hospital, JL Marg, New Delhi, India
}

\begin{abstract}
Background: Nosocomial septicemia due to extended spectrum beta-( $\beta$ )-lactamase (ESBL) producing Klebsiella pneumoniae and Escherichia coli are a therapeutic challenge due to resistance. Knowledge of disease burden and resistance patterns is required for proper and timely management. We report the prevalence and antimicrobial susceptibility of ESBL producing E. coli and $K$.pneumoniae from septicemia at a tertiary care hospital.

Methodology: A total of 2,870 blood samples of suspected cases of septicemia were studied between January and December 2009 . Antimicrobial susceptibility was determined by Kirby Bauer's disc diffusion method and MICs for imipenem, meropenem, and ertapenem were determined using the E-test. All isolates of E. coli and $K$. pneumoniae were tested for ESBL production by E-test method.

Results: Forty-one (70.7\%) K. pneumoniae isolates and ten (41.7\%) E. coli isolates were ESBL producers. Two (5\%) of ESBL producing K. pneumoniae isolates, but no $E$. coli isolates, were resistant to carbapenems. In vitro, all ESBL producers were sensitive to tigecycline.

Conclusion: Our data indicated that the prevalence of ESBL-producing E. coli and K. pneumonia strains isolated from blood cultures from hospitalized patients is high. ESBL-producing organisms were found to be more susceptible to meropenem than to imipenem and ertapenem. Tigecycline is active against all the ESBL or multidrug resistant (MDR) E. coli and Klebsiella spp. isolates.
\end{abstract}

Key words: nosocomial; bacteremia; extended spectrum beta-lactamase

J Infect Dev Ctries 2010; 4(8):517-520.

(Received 11 November 2009 - Accepted 07 April 2010)

Copyright $\odot 2010$ Taneja et al. This is an open-access article distributed under the Creative Commons Attribution License, which permits unrestricted use, distribution, and reproduction in any medium, provided the original work is properly cited.

\section{Introduction}

Extended spectrum beta-( $\beta$ )-lactamase (ESBL) producing strains of Enterobacteriaceae have now emerged as a major problem in hospitalized patients. These organisms are responsible for a variety of infections, such as septicemia, urinary tract infection, hospital-acquired pneumonia, intra-abdominal abscess, brain abscess, and device-related infections [1]. Among all types of nosocomial infections, the nosocomial blood-stream infection (BSI) creates a serious health problem in hospitals all over the world. Escherichia coli and Klebsiella pneumoniae are major nosocomial pathogens causing intra-abdominal infection, urinary tract infection, and primary bacteremia. ESBL producers show resistance to cephalosporins and aztreonam, and often carry resistance to aminoglycosides and other drugs, but they remain susceptible to carbapenems [2]. Unfortunately, there is now an increasing occurrence of carbapenem resistance in Enterobacteriaceae. In this context, clinical response to new antibiotics with good in vitro activity against ESBL producers, such as tigecycline, needs to be evaluated. This study investigated the nosocomial BSI in the adult intensive care units and prevalence of extended spectrum $\beta$ lactamase producers among E. coli and Klebsiella sp. It also studied the in vitro activity of imipenem (IPM), meropenem (MEM), ertapenem (ETP), and tigecycline (TGC) against ESBL-producing common pathogens isolated from patients in intensive care.

\section{Material and methods}

All adult patients admitted at different intensive care units (ICUs) of GB Pant Hospital (coronary care unit, CCU; cardiothoracic surgery ICU; neurological ICU; general ICU; and neurosurgical ICU) were included in the study during January 2009 to December 2009. Surveillance of these patients was conducted by the laboratory-based ward Liaison method to identify BSI. BSI were defined as a bloodstream infections documented by at least one blood culture positive from a patient with systemic inflammatory response syndrome (e.g. fever, 
Table 1. Susceptibility patterns of ESBL-producing organisms

\begin{tabular}{|l|l|l|}
\hline Antimicrobial Agent & E. coli $\mathrm{n}=10(\%)$ & K. pneumonia $\mathrm{n}=41(\%)$ \\
\hline Imipenem & $10(100)$ & $39(95.1)$ \\
\hline Meropenem & $10(100)$ & $39(95.1)$ \\
\hline Ertapenem & $10(100)$ & $39(95.1)$ \\
\hline Amikacin & $7(70)$ & $15(36.6)$ \\
\hline Gentamicin & $3(30)$ & $5(12.2)$ \\
\hline $\begin{array}{l}\text { Piperacillin- } \\
\text { Tazobactam }\end{array}$ & $6(60)$ & $11(26.8)$ \\
\hline $\begin{array}{l}\text { Ticarcillin- } \\
\text { Clavulanic acid }\end{array}$ & $(0)$ & $(0)$ \\
\hline Ofloxacin & $3(30)$ & $9(21.9)$ \\
\hline Cefepime & $(0)$ & $(0)$ \\
\hline Cefotaxime & 0 & 0 \\
\hline Tigecycline & $10(100)$ & $41(100)$ \\
\hline
\end{tabular}

tachycardia, tachypnea, and leukocytosis) [3]. All the blood samples were processed by the clinical microbiology laboratory in GB Pant Hospital. The pathogens were identified by conventional biochemical procedures [4]. All isolates were screened phenotypically for ESBL production by Etest (AB Biodisk, Solna, Sweden). Minimum inhibitory concentration (MIC) of ESBL-producing strains to cefotaxime was also determined by E-test. ESBL-producing $E$. coli and $K$. pneumoniae were included in the study. Only one non-repeat isolate from each bacteremic episode was included in the analysis. The antibiotic susceptibility of each isolate was determined by the disc diffusion method, employing the criteria of the Clinical and Laboratory Standards Institute (CLSI) guidelines [5]. The antibiotics included in the susceptibility test were cefotaxime $(30 \mu \mathrm{g})$, cefepime $(30 \mu \mathrm{g})$, ofloxacin $(5$ $\mu \mathrm{g})$, piperacillin-tazobactum $(100 / 10 \mu \mathrm{g})$, ticarcillinclavulanic acid $(75 / 10 \mu \mathrm{g})$, amikacin $(30 \mu \mathrm{g})$, gentamicin $(10 \mu \mathrm{g})$, imipenem IPM $(10 \mu \mathrm{g})$ (HiMedia, Mumbai, India), meropenem MEM (10 $\mu \mathrm{g})$ (Becton Dickinson, Sparks, MD, USA), ertapenem ETP $(10 \mu \mathrm{g})$ (Becton Dickinson, Sparks, MD, USA), and tigecycline TGC $(10 \mu \mathrm{g})$ (Becton Dickinson, Sparks, MD, USA). Isolates were considered resistant to MEM and IPM if the zone of inhibition was $\leq 13$ $\mathrm{mm}$, intermediate when the zone was $14-15 \mathrm{~mm}$, and sensitive when the zone was $\geq 16 \mathrm{~mm}$, per CLSI guidelines [5]. For ETP, isolates were considered resistant when the zone diameter was $\leq 15 \mathrm{~mm}$, intermediate when the zone diameter was $16-18 \mathrm{~mm}$, and sensitive when the zone diameter was $\geq 19 \mathrm{~mm}$. For TGC, isolates were considered resistant when the zone diameter was $\leq 14 \mathrm{~mm}$, intermediate when the zone diameter was $15-18 \mathrm{~mm}$, and sensitive when the zone diameter was $\geq 19 \mathrm{~mm}$. The MIC of ETP, IPM, and MEM was also recorded for all the isolates by the E-test (AB Biodisk, Solna, Sweden). The MIC breakpoints for IMP, MEM, and ETP were taken per CLSI guidelines [5]. For IPM and MEM, isolates were considered sensitive if the MIC was $\leq 4 \mu \mathrm{g} / \mathrm{mL}$, intermediate if the MIC was $8 \mu \mathrm{g} / \mathrm{mL}$, and resistant if the MIC was $\geq 16 \mu \mathrm{g} / \mathrm{mL}$. Isolates were considered sensitive to ertapenem if the MIC was $\leq 2 \mu \mathrm{g} / \mathrm{mL}$, intermediate if the MIC was $4 \mu \mathrm{g} / \mathrm{mL}$ and resistant if the MIC was $\geq 8 \mu \mathrm{g} / \mathrm{mL}$. Two control organisms, $E$. coli ATCC 25922 and K. pneumoniae ATCC 700603, were inoculated in each set of tests for quality control.

\section{Results}

Out of 2,870 blood cultures obtained from suspected cases of septicemia, 244 (8.5\%) yielded growth of microorganisms. The total number of pathogenic isolates was 253, of which six were Candida spp. and 247 were bacterial isolates. Nine samples showed polymicrobial growth of Gramnegative bacteria. The Gram-negative bacteria most commonly isolated from blood cultures were $K$. pneumonia 64 (26.2\%), Acinetobacter spp. 63 (25.8 $\%)$, Escherichia coli 26 (10.6\%), Citrobacter spp.15 (6.1\%), Pseudomonas spp. 24 (9.8\%), Enterobacter spp. 9 (3.7 \%), and Proteus mirabilis 1 (0.4\%). The Gram-positive bacteria isolated were Staphylococcus aureus 14 (5.7 \%), Enterococcus spp. 26 (10.6\%), Streptococcus spp. 4 (1.6\%), and coagulase negative staphylococcus $1(0.4 \%)$.

All strains of $E$. coli and $K$. pneumoniae were tested for ESBL production. ESBL was detected in 
$41(64 \%)$ isolates of K. pneumoniae and 10 (38.5\%) isolates of E. coli. All ESBL isolates had MIC $>8$ $\mu \mathrm{g} / \mathrm{mL}$ to cefotaxime. The susceptibility data of the ESBL-producing $E$. coli and $K$. pneumoniae are summarized in Table 1. Tigecycine and carbapenems showed sensitivity of $100 \%$ and $95 \%$, respectively, by disc diffusion. Two Klebsiella spp. isolates were resistant to all the carbapenems tested, with MICs for IPM, MEM, and ETP of 8,4 , and $8 \mu \mathrm{g} / \mathrm{mL}$ respectively. A follow-up of the cases of septicaemia due to ESBL-producing bacteria revealed that four out of 51 patients $(7.8 \%$ ), including two ESBL cases resistant to carbapenems, expired during their hospital stay and the rest were discharged after recovery.

\section{Discussion}

Extended spectrum $\beta$-lactamase producing Gramnegative bacteria, particularly Klebsiella pneumoniae and Escherichia coli, are now widespread nosocomial pathogens causing bacteremia. The most frequent isolates in the present study were $K$. pneumoniae (26.2\%) and Acinetobacter (25.8\%). Similar results are reported by previous studies from India [6,7]. The current study shows prevalence of ESBL-producing E. coli and K. pneumoniae as $10(41.7 \%)$ and 41 $(70.7 \%)$, respectively. Other recent studies from India have reported a prevalence of ESBL production ranging from $41.0-63.6 \%$ in E. coli and $40-66.7 \%$ in $K$. pneumoniae [7,8]. Carbapenems are effective alternative antibiotics against ESBL-producing bacteria. The carbapenems tested in the present study showed high activity against all strains of $E$. coli and $K$. pneumoniae with the exception of two $K$. pneumoniae isolates. Resistance in ESBL-producing members of Enterobacteriaecea has been reported as $3-6 \%$ to carbapenems [9].

Tigecycline has a bacteriostatic mode of action against a broad spectrum of both aerobic and anaerobic Gram-positive (including methicillinresistant Staphylococcus aureus and vancomycin resistant enterococci) organisms and Gram-negative organisms [10]. Regarding ESBL-producing Enterobacteriaceae, in vitro susceptibility to tigecycline for E. coli and K. pneumoniae has been reported as $99.8 \%$ and $92.3 \%$, respectively [10,11]. All ESBL producers in the present study showed sensitivity to tigecycline. E. coli, and $K$. pneumoniae isolates showed a sensitivity of $75 \%$ and $35 \%$ to amikacin, respectively. Similar findings observed by Winokur et al. [12] showed that $80-87 \%$ of ESBL strains were resistant to ofloxacin. Regional studies have reported the emergence of fluoroquinolone coresistance in ESBL-producing organisms [13]. All the $E$. coli and $K$. pneumoniae ESBL-producing isolates were resistant to cefotaxime and cefepime

In conclusion, our data indicated the high prevalence of ESBL-producing $E$. coli and $K$. pneumonia strains isolated from blood cultures from hospitalized patients. ESBL-producing organisms were found to be more susceptible to meropenem than to imipenem and ertapenem; however, the MICs of ertapenem and imipenem were well within sensitive levels. Tigecycline is active against almost all the ESBL or MDR E. coli and Klebsiella spp. isolates. Acquiring ESBL-producing bacteria can be prevented by adherence to infection control practices in the hospital, such as the removal of medical devices as soon as possible and adherence to sterile techniques when performing procedures. Tigecycline holds promise as an alternative choice of therapy for infections caused by ESBL-producing isolates, keeping in mind the emerging carbapenem resistance.

\section{References}

1. Behera B, Mathur P, Das A, Kapil A (2009) Ertapenem susceptibility of extended spectrum beta-lactamaseproducing Enterobacteriaceae at a tertiary care centre in India. Singapore Med J 50: 628-632.

2. Paterson DL, Bonomo RA (2005) Extended-spectrum Blactamases: a clinical update. Clin Microbiol Rev 18: 657686.

3. Russell, JA (2006) Management of sepsis. N Engl J Med 355: 1699-1713.

4. Collee JG, Fraser AG, Marmion BP, Simmons A (1996) In: Mackie and McCartney Practical Medical Microbiology, 14th ed. Churchill Livingstone, London. pp. 385-404.

5. Clinical and Laboratory Standards Institute (2008) Performance standards for antimicrobial susceptibility testing, 18th informational supplement. CLSI document no. M100-S18.

6. Kapoor, H, Sumathi, M, Aggarwal P, Jain, SD, Kaur, J (2000) Spectrum of bacterial isolates in high risk areas of a tertiary care hospital: 3 year study. Indian J Med Microbiol 18: 166-169.

7. Jain A, Roy I, Gupta MK, Kumar M, Agarwal SK (2003) Prevalence of extended spectrum beta-lactamase producing Gram-negative bacteria in septicaemic neonates in a tertiary care hospital. J Med Microbiol 52: 421-5.

8. Goyal, A, Prasad KN, Prasad A, Gupta S, Ghoshal U, Ayyagari A (2009) Extended spectrum $\beta$-lactamases in Escherichia coli \& Klebsiella pneumoniae \& associated risk factors. Indian J Med Res 129: 695-700.

9. Gupta E, Mohanty S, Sood S, Dhawan B, Das BK, Kapil A. (2006) Emerging resistance to carbapenems in a tertiary care hospital in north India. Indian J Med Res 124(1): 95-98.

10. Kelesidis T, Karageorgopoulos DE, Kelesidis I, Falagas ME (2008) Tigecycline for the treatment of multidrug-resistant Enterobacteriaceae: a systematic review of the evidence 
from microbiological and clinical studies. J Antimicrob Chemother 62: 895-904.

11. Jamal WY, Al Hashem G, Khodakhast F, Rotimi VO (2009) Comparative in vitro activity of tigecycline and nine other antibiotics against Gram-negative bacterial isolates, including ESBL-producing strains. J Chemother 21: 261266.

12. Winokur PL, Canton R, Casellas JM, Legakis N (2001) Variations in the prevalence of strains expressing an extending-spectrum $\beta$ - lactamase phenotype and characterization of isolates from Europe, the Americas, and the Western Pacific region. Clin Infect Dis 32 Supp 2: S94103.

13. Spanu T, Luzzaro F, Perilli M, Amicosante G, Toniolo A, Fadda G; Italian ESBL Study Group (2002) Occurrence of extended-spectrum beta-lactamases in members of the family Enterobacteriaceae in Italy: implications for resistance to beta-lactams and other antimicrobial drugs. Antimicrob Agents Chemother 46: 196-202.

\section{Corresponding author \\ Dr Juhi Taneja \\ Senior Resident \\ GB Pant Hospital \\ JL Marg, New Delhi \\ India \\ E mail-drjuhitaneja@gmail.com}

Conflict of interests: No conflict of interests is declared. 